\title{
Prediction of lactate threshold using the modified Conconi test in distance runners
}

\author{
M Kjertakov', M Dalip ${ }^{2}$, R Hristovski ${ }^{3}$, Y Epstein ${ }^{4,5}$ \\ ${ }^{1}$ Independent Researcher \\ ${ }^{2}$ Faculty of Physical Education, State University of Tetova, Tetovo, Republic of Macedonia \\ ${ }^{3}$ Faculty of Physical Education, Sport and Health, Ss. Cyril and Methodius University, \\ Skopje, Republic of Macedonia \\ ${ }^{4}$ Heller Institute of Medical Research, Sheba Medical Center, Tel-Hashomer, Israel \\ ${ }^{5}$ Sackler Faculty of Medicine, Tel Aviv University, Tel Aviv, Israel
}

Received: September 19, 2015

Accepted: March 10, 2016

\begin{abstract}
This study aimed to examine the validity of the modified Conconi test (CT) to predict lactate threshold (LT) during running. Twelve distance runners randomly performed the modified CT and the incremental test in which LT was determined directly by measuring blood lactate (BLa). Mean values of heart rate (HR) and running speed (RS) at heart rate deflection point $\left(\mathrm{HR}_{\mathrm{DP}}\right)$ obtained through the modified $\mathrm{CT}$ were compared with those at LT. Subsequently, the runners who showed a $\mathrm{HR}_{\mathrm{DP}}$ in the modified CT performed a 30-min prolonged exercise test (PET) at a RS corresponding to $\mathrm{HR}_{\mathrm{DP}}$. During this test, the kinetics of $\mathrm{BLa}$ and $\mathrm{HR}$ were analyzed to determine whether a steady state in these variables could be attained. We succeeded in identifying $\mathrm{HR}_{\mathrm{DP}}$ in nine of our runners, whereas the remaining three runners showed entirely linear HR response. In those nine runners, no significant difference was found between $\mathrm{HR}$ and RS at $\mathrm{HR}_{\mathrm{DP}}$ and those at $\mathrm{LT}$. Significant correlation was found between $\mathrm{HR}$ at $\mathrm{HR}_{\mathrm{DP}}$ and $\mathrm{HR}$ at LT $(r=0.84, p=0.005)$, but RS at $\mathrm{HR}_{\mathrm{DP}}$ was not significantly correlated with $\mathrm{RS}$ at $\mathrm{LT}(r=0.63, p=0.07)$. All nine runners were able to complete the PET with steady state conditions being achieved for both BLa and HR. In conclusion, these findings indicate that the modified $\mathrm{CT}$ has a potential to be used as an alternative method for assessment of $\mathrm{LT}$ in distance runners presenting a $\mathrm{HR}_{\mathrm{DP}}$.
\end{abstract}

Keywords: heart rate deflection point, maximal deviation method, endurance athletes, distance running, exercise test

The lactate threshold (LT), i.e., the level of exercise above which blood lactate (BLa) concentration sharply increases (6) has become commonly measured physiological parameter in distance runners (30). Its use for assessing endurance capacity, prescribing training intensity and predicting endurance performance in these types of athletes has been well documented $(2,21)$. Conventionally, LT is determined through the analysis of BLa curve obtained in the laboratory using an incremental treadmill test protocol. To improve the precision of LT identification on the BLa curve, several methods have been proposed in the last decades $(3,6)$. Currently, one of the most commonly used methods is the maximal deviation method (Dmax) proposed by Cheng et al. (12). Dmax is an objective and individual method that estimates the LT by identifying the point on the lactate-exercise intensity regression curve that yields the maximal distance to the straight line formed by the first and the last point of the curve (12).

Corresponding author: Metodija Kjertakov

Thorndon Dr. 8/8, Melbourne 3021, Australia

Phone: +61 415655107; E-mail: metodijakjertakov@gmail.com 
Although distance runners may benefit from determination of LT, the aforementioned testing procedure is costly and it is therefore not readily available. As a consequence of this, many coaches and runners are left to rely on alternative methods for LT determination. Among these methods, the most popular appears to be the one proposed by Conconi et al., which is simply based on the analysis of the heart rate (HR) response to an incremental running test (13). These researchers reported that the HR-running speed (RS) relationship is linear from low to submaximal speeds and curvilinear from submaximal to maximal speeds. Furthermore, they have shown that the point at which HR departs from linearity, labeled as heart rate deflection point $\left(\mathrm{HR}_{\mathrm{DP}}\right)$, coincided with the start of a sharp accumulation of BLa. From these findings, Conconi et al. (13) suggested that the $\mathrm{RS}$ at the $\mathrm{HR}_{\mathrm{DP}}$ can be used to predict LT in runners. The $\mathrm{HR}_{\mathrm{DP}}$ concept was later also applied to other endurance sports such as cycling, rowing and swimming $(11,15)$.

The validity of the Conconi method has been examined in many studies but they have produced contradictory results. Whereas some researchers $(1,9,29)$ have succeeded to duplicate the findings of Conconi et al. (13), others $(18,22,23)$ reported that $\mathrm{HR}_{\mathrm{DP}}$ could not be always detected, and when detected, it did not necessarily coincide with LT. Therefore, doubts have been raised about the usefulness of this method in the assessment of LT $(4,20)$.

According to Conconi et al. (14), at least some of the reported unsuccessful attempts to validate their method by other researchers can be attributed to methodological problems associated with the testing protocol. To overcome these problems, in 1996, the authors have modified the original protocol of the test and provided detailed information on how to perform, analyze and avoid experimental conditions that could negatively influence its outcome (14). The differences between the new protocol and the original one (13) are as follows: (1) speed increases in a ramp-like fashion (uniformly) rather than in a step-like way and (2) increases in speed are based on time rather than distance.

Despite the fact that the modified version of the so-called Conconi test (CT) has been introduced two decades ago, to our knowledge, only one study has attempted to assess its validity against the conventional incremental running test in which LT was directly measured (26). In this study, McGehee et al. (26) found that $H R$ at $H_{D P}$ did not differ significantly from the HR corresponding to the directly measured LT, but RS at $\mathrm{HR}_{\mathrm{DP}}$ was found to be significantly greater than that at "true" LT. In other words, these authors showed that the modified CT accurately predicted HR at LT, but did not accurately predict RS at LT. The inconsistency in the findings of this study suggests that further research is needed.

The main aim of this study was to re-examine the validity of the modified CT to predict HR and RS at LT using the conventional incremental test in a group of well-trained distance runners. Another approach to validate the modified CT in this study was the analysis of the physiological responses of these runners to prolonged exercise test (PET) at an RS corresponding to $\mathrm{HR}_{\mathrm{DP}}$.

\section{Materials and Methods}

\section{Participants}

Twelve well-trained distance runners (11 male and 1 female) volunteered to participate in this study. Their mean \pm SD age, height, weight and body mass index were $24.1 \pm 3.9$ years, $177.3 \pm 5.7 \mathrm{~cm}, 63.1 \pm 6.5 \mathrm{~kg}$ and $20.2 \pm 1.3 \mathrm{~kg} / \mathrm{m}^{2}$, respectively. All runners had at least 1 year of competitive experience at national and international level and run $\geq 60 \mathrm{~km}$ per week 
at the time of this study. The study was approved by the Institutional Review Board of Faculty of Physical Education at the State University of Tetova (Approval No. 02-3193) and a written informed consent was obtained from all participants. The runners were asked to maintain their usual diet throughout the study and to avoid intensive or prolonged exercise the day before each test. They were also instructed to report for testing in a rested and well-hydrated state, at least $3 \mathrm{~h}$ postprandial and not to have consumed alcohol, caffeine or any stimulating substances for a minimum of $24 \mathrm{~h}$. In addition, prior to commencement of the testing, the runners were familiarized with the testing environment and with the equipment that was used subsequently.

\section{Experimental design}

After a familiarization session, all the runners randomly performed the modified CT and the conventional incremental test in which LT was determined directly by measuring BLa concentrations (i.e., the criterion test). Mean values of $\mathrm{HR}$ and $\mathrm{RS}$ at $\mathrm{HR}_{\mathrm{DP}}$ obtained through the modified CT were then statistically compared with those at LT to determine the accuracy of the modified CT in predicting LT. Subsequently, the runners who showed a $\mathrm{HR}_{\mathrm{DP}}$ in the modified CT performed a PET at an RS corresponding to $\mathrm{HR}_{\mathrm{DP}}$. During this test, the kinetics of BLa and HR were analyzed to determine whether or not a steady state in these variables could be attained. Furthermore, the values of BLa and HR observed during the test were compared to those from the criterion test.

\section{Procedures}

All tests were performed on a motorized treadmill (Technogym, Gambettola, Italy) with the gradient set at $1 \%$, and at the same time in the afternoon on separate days. All testing was completed for each runner within a 2-week period, with a time interval of at least $72 \mathrm{~h}$ between each test. Through all testing, the air temperature in the testing room was maintained between $19^{\circ} \mathrm{C}$ and $22{ }^{\circ} \mathrm{C}$ and the runners were fan-cooled. The runners also wore the same running shoes and lightweight running kit for all tests. Each test was preceded by a 20 -min warm-up at a velocity corresponding to $\sim 65 \%$ of the runners' real maximal heart rate $\left(H R_{\max }\right)$, which was determined during the familiarization session following the testing protocol described elsewhere (30). HR was recorded continuously every $5 \mathrm{~s}$ during the tests using a Polar RS 800 heart rate monitor (Polar Electro OY, Kempele, Finland).

The $\mathrm{HR}_{\mathrm{DP}}$ was determined according to the protocol of Conconi et al. (14). Briefly, the runners started to run at $9 \mathrm{~km} / \mathrm{h}$ and the speed was increased by $0.5 \mathrm{~km} / \mathrm{h}$ every minute until volitional exhaustion. According to the authors, such a gradual increment in RS throughout the test is necessary in order to elicit the increase in HR by less than 8 beats/min each minute, if the test is considered to be successful (14). The HRs which were recorded at the end of each minute of the test were used to generate $\mathrm{HR}-\mathrm{RS}$ plots from which $\mathrm{HR}_{\mathrm{DP}}$ was identified by visual analysis (Fig. 1).

For LT determination, an initial test speed was set at $9 \mathrm{~km} / \mathrm{h}$, with increments of $1 \mathrm{~km} / \mathrm{h}$ every $4 \mathrm{~min}$ until volitional exhaustion. Blood samples were obtained from a fingertip at rest, after the warm-up and during the 30-s breaks between the exercise stages and immediately analyzed using a hand-held lactate analyzer (Lactate Scout, Senslab $\mathrm{GmbH}$, Leipzig, Germany). The validity of this device has been previously confirmed (31). The data obtained from the test were entered in a computer software (Lactate-R, designed by Newell et al.) through which LT was calculated by applying the Dmax method (28). 


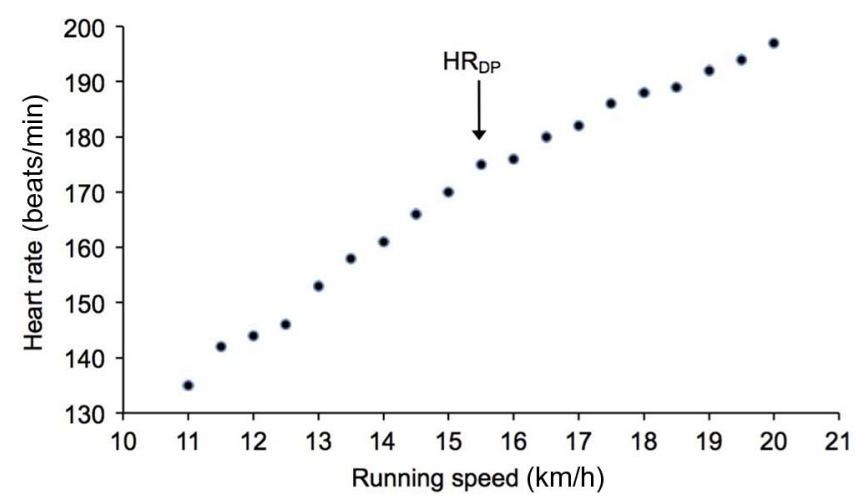

Fig. 1. Example of determination of heart rate deflection point $\left(\mathrm{HR}_{\mathrm{DP}}\right)$ in one runner from the relationship between heart rate (HR) and running speed (RS)

Subsequently, the runners who showed a $\mathrm{HR}_{\mathrm{DP}}$ in the modified CT performed a 30-min PET at an RS corresponding to $\mathrm{HR}_{\mathrm{DP}}$. Blood samples were obtained from a fingertip at rest, after the warm-up, during the $30 \mathrm{~s}$ breaks after every $5^{\text {th }}$ minute throughout the test and at the end of the test and immediately analyzed using the aforementioned lactate analyzer. In this study, steady state was defined as the exercise intensity at which the activity of the physiological variables did not increase significantly during the last $10 \mathrm{~min}$ of the test, as proposed by Hofmann et al. (19).

\section{Statistical analyses}

Data are expressed as mean \pm standard deviation. The normality of the data distribution was verified by the Shapiro-Wilk test. Within group, $t$-test was applied to compare data sets between the modified CT and the criterion test. The relationship between the variables at $\mathrm{HR}_{\mathrm{DP}}$ and those at LT was determined using Pearson product-moment correlation analysis. One-way analysis of variance for repeated measures was used to compare the changes in physiological variables during the 30-min PET. When statistical significance was found, a Tukey post hoc test was performed to locate differences among the time points. Statistical significance was set at $p<0.05$. Statistical analyses were performed using STATISTICA software (version 10.0, StatSoft).

\section{Results}

Table I shows the maximal values of HR and RS obtained during the modified CT and the criterion test. There was no significant difference between the $\mathrm{HR}_{\max }$ attained in the modified $\mathrm{CT}$ and that attained in the criterion test. However, the runners attained a significantly higher maximal running speed $\left(\mathrm{RS}_{\max }\right)$ in the modified CT compared to the criterion test $(p=0.01)$.

For the modified $\mathrm{CT}$, the mean $( \pm \mathrm{SD})$ increase in HR between the exercise stages was $3.0 \pm 1.6$ beats $/ \mathrm{min}$. The $\mathrm{HR}_{\mathrm{DP}}$ could not be identified in three runners and therefore the data of those runners were excluded from the final analysis. The mean data for HR and RS at $\mathrm{HR}_{\mathrm{DP}}$ and at $\mathrm{LT}$ of the remaining nine runners are presented in Table II. In these runners, $\mathrm{HR}_{\mathrm{DP}}$ occurred at $91.6 \%$ and $80.8 \%$ of $\mathrm{HR}_{\max }$ and $\mathrm{RS}_{\max }$, respectively. Furthermore, LT occurred at $90.5 \%$ and $81.6 \%$ of $\mathrm{HR}_{\max }$ and $\mathrm{RS}_{\max }$, respectively. The mean $( \pm \mathrm{SD})$ value for $\mathrm{BLa}$ at LT was $2.56 \pm 0.53 \mathrm{mmol} / \mathrm{L}$. The RS at which $\mathrm{HR}_{\mathrm{DP}}$ occurred was not significantly different from that at LT. No significant difference was found between $\mathrm{HR}$ at $\mathrm{HR}_{\mathrm{DP}}$ and $\mathrm{HR}$ at 
Table I. Maximal values obtained during the modified CT and the criterion test

\begin{tabular}{|l|c|c|c|}
\hline Variable & Modified CT & Criterion test & $p$ \\
\hline RS $(\mathrm{km} / \mathrm{h})$ & $19.42 \pm 0.7$ & $19.0 \pm 0.9$ & 0.01 \\
\hline HR (beats/min) & $194.0 \pm 8.7$ & $192.8 \pm 9.7$ & 0.26 \\
\hline BLa $(\mathrm{mmol} / \mathrm{L})$ & - & $11.7 \pm 2.0$ & - \\
\hline
\end{tabular}

$\mathrm{RS}=$ running speed $\mathrm{HR}=$ heart rate $; \mathrm{BLa}=$ blood lactate

Table II. HR and RS values at $\mathrm{HR}_{\mathrm{DP}}$ and $\mathrm{LT}$

\begin{tabular}{|l|c|c|c|c|}
\hline & \multicolumn{2}{|c|}{ HRDP } & \multicolumn{2}{c|}{ LT } \\
\hline Runner & RS (km/h) & HR (beats/min) & RS (km/h) & HR (beats/min) \\
\hline 1 & 15.5 & 175 & 16.1 & 175 \\
\hline 2 & 16.0 & 183 & 16.7 & 183 \\
\hline 3 & 17.0 & 175 & 16.4 & 167 \\
\hline 4 & 16.0 & 180 & 15.0 & 181 \\
\hline 5 & 16.0 & 184 & 16.2 & 184 \\
\hline 6 & 16.0 & 190 & 15.6 & 189 \\
\hline 7 & 14.5 & 174 & 14.8 & 177 \\
\hline 8 & 15.5 & 186 & 16.6 & 178.3 \\
\hline 9 & 14.5 & 176 & 14.7 & 6.3 \\
\hline SD & 15.6 & 5.7 & & 15.8 \\
\hline
\end{tabular}

$\mathrm{RS}=$ running speed; $\mathrm{HR}=$ heart rate; $\mathrm{HR}_{\mathrm{DP}}=$ heart rate deflection point; $\mathrm{LT}=$ lactate threshold

LT either. Significant correlation was found between HR at $\mathrm{HR}_{\mathrm{DP}}$ and HR at LT $(r=0.84$, $p=0.005)$, but $\mathrm{RS}$ at $\mathrm{HR}_{\mathrm{DP}}$ was not significantly correlated with $\mathrm{RS}$ at $\mathrm{LT}(r=0.63$, $p=0.07)$.

The mean $( \pm \mathrm{SD}) \mathrm{BLa}$ and $\mathrm{HR}$ values during the PET at RS corresponding to $\mathrm{HR}_{\mathrm{DP}}$ for the nine runners who demonstrated a deflection point in HR-RS relationship in the modified CT are presented in Fig. 2. All runners were able to complete the test, and since the BLa and HR values did not increase significantly between the $20^{\text {th }}$ and $30^{\text {th }}$ minute of test, it was accepted that steady state conditions were achieved for both variables. Furthermore, there were no significant differences between the mean $( \pm S D)$ values for BLa and HR measured at the end of the PET and those at LT, derived from the criterion test. 
(a)
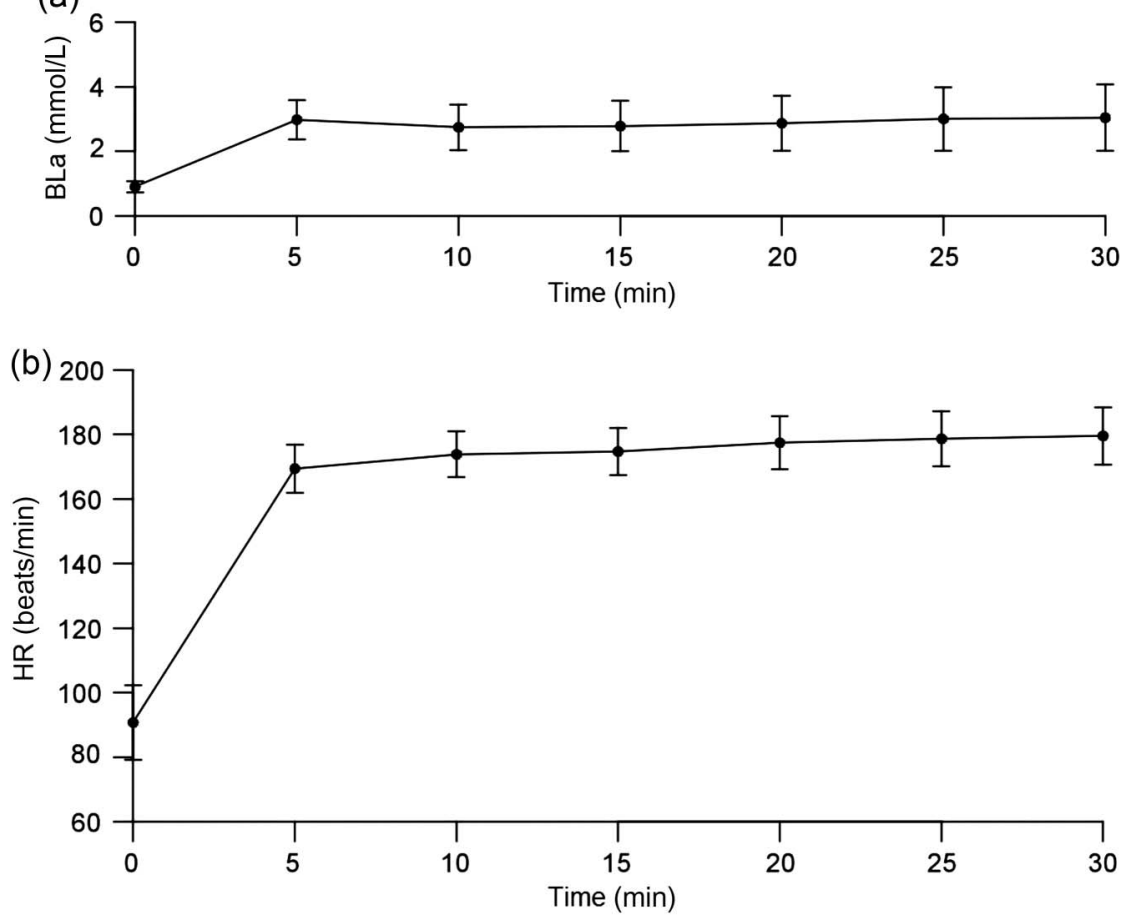

Fig. 2. Blood lactate (a) and heart rate (b) kinetics during prolonged exercise test at a running speed corresponding to heart rate deflection point $\left(\mathrm{HR}_{\mathrm{DP}}\right)$

\section{Discussion}

This study aimed to examine the validity of the modified CT to predict LT in a group of welltrained distance runners. The design of our study was very similar to those previously reported by Bourgois and Vrijens (8) and Bourgois et al. (7) who also assessed the validity of the modified $\mathrm{CT}$, but during rowing and cycling.

In this study, we succeeded in identifying $\mathrm{HR}_{\mathrm{DP}}$ in nine of our runners, whereas the remaining three runners showed entirely linear HR response. These results support the findings of other investigators who documented that $\mathrm{HR}_{\mathrm{DP}}$ could not always be observed during an incremental exercise test even when the modified CT protocol was applied $(24,25)$. Bourgois and Vrijens (8) and Bourgois et al. (7), on the other hand, were able to detect $\mathrm{HR}_{\mathrm{DP}}$ in all of their subjects who were also submitted to the modified CT. The percentage of $\mathrm{HR}_{\max }$ (91\%) at which $\mathrm{HR}_{\mathrm{DP}}$ occurred in our runners is comparable to the values reported in previous studies with athletes of various sports, ranging from $88 \%$ to $92 \%(5,10,25)$. To identify $\mathrm{HR}_{\mathrm{DP}}$, we used manual graphing and visual analysis, which is in agreement with the aforementioned studies $(7,8)$. Although this approach for identification of $\mathrm{HR}_{\mathrm{DP}}$ may not be the most accurate, it is the most practical.

The main finding of this study is that there were no significant differences between the mean $\mathrm{HR}$ and $\mathrm{RS}$ at $\mathrm{HR}_{\mathrm{DP}}$ and those at LT. Furthermore, the correlation between $\mathrm{HR}$ at $\mathrm{HR}_{\mathrm{DP}}$ and HR at LT was found to be high $(r=0.84, p=0.005)$, though $\mathrm{RS}$ at $\mathrm{HR}_{\mathrm{DP}}$ did not significantly correlate with RS at LT $(r=0.63, p=0.07)$. Recently, McGehee et al. (26) also 
attempted to validate the accuracy of the modified CT for prediction of LT during running, using a similar criterion test as in our study. Investigating 27 distance runners and triathletes, they found that $\mathrm{HR}$ at $\mathrm{HR}_{\mathrm{DP}}$ did not differ significantly from that at LT, which is in line with our data; however, in contrast to our findings, $\mathrm{RS}$ at $\mathrm{HR}_{\mathrm{DP}}$ was found to be significantly greater than that at LT $(p<0.05)$. They also found significant relationship between HR at $\mathrm{HR}_{\mathrm{DP}}$ and HR at LT $(r=0.78, p<0.001)$ and, unlike in our study, between $\mathrm{RS}$ at $\mathrm{HR}_{\mathrm{DP}}$ and $\mathrm{RS}$ at LT $(r=0.72, p<0.001)$. From these observations, the authors concluded that the modified CT can be used to accurately estimate HR at LT. We have no possible explanation for the inconsistency in the findings between ours and the study of McGehee et al. (26). Bodner et al. (5) came to a same conclusion as McGehee et al. (26) following the investigation of 22 cyclists during bicycling ergometry. These investigators compared HR, $\mathrm{VO}_{2}$ and power output associated with $\mathrm{HR}_{\mathrm{DP}}$, measured by using the modified $\mathrm{CT}$ and those at ventilatory threshold (VT), a parameter which has been accepted as a valid estimator of LT. No significant differences were noted for $\mathrm{HR}$ and $\mathrm{VO}_{2}$ at $\mathrm{HR}_{\mathrm{DP}}$ and at VT, but power output at $\mathrm{HR}_{\mathrm{DP}}$ was significantly different from power output at $\mathrm{VT}(p<0.01)$. Significant relationships were found between $\mathrm{HR}_{\mathrm{DP}}$ and VT for HR $(r=0.92, p<0.001)$, $\mathrm{VO}_{2}(r=0.72, p<0.001)$ and power output $(r=0.77, p<0.001)$. Similar findings have been presented by Cabo et al. (10), Erdogan et al. (16) and Mikulic et al. (27) for oarsmen and by Fabre et al. (17) for cross-country skiers, using the modified CT. However, not all studies that dealt with the modified CT have reported results in favor of this test $(7,8)$. Nevertheless, this study clearly shows that the modified CT accurately predicted LT in the runners presenting a $\mathrm{HR}_{\mathrm{DP}}$.

This statement is further supported by the results of the PET. Indeed, all nine runners were able to complete the 30-min PET at a work load corresponding to $\mathrm{HR}_{\mathrm{DP}}$ with a steadystate condition being achieved for both BLa and HR. Furthermore, the mean values for BLa and HR obtained at the end of the PET were not significantly different from those at LT. These findings contradict the observations reported by Bourgois and Vrijens (8) and Bourgois et al. (7) according to which $\mathrm{HR}_{\mathrm{DP}}$ overestimates the sustainable exercise intensity. It should be mentioned, however, that these investigators adopted different criteria to define the steady state comparing with the criteria used in our study (see Materials and Methods section). In their studies, a steady-state condition for BLa, the only variable that they have analyzed during the PET, was defined as an increase in BLa concentration of less than $1 \mathrm{mmol} / \mathrm{L}$ between the $10^{\text {th }}$ and the $30^{\text {th }}$ minute of the test. In the first study (8), the authors found a BLa steady state in only 1 out of 10 rowers who performed a 30-min PET on a rowing ergometer at power output corresponding to $\mathrm{HR}_{\mathrm{DP}}$. Three other rowers also succeeded in completing the PET but failed to attain the BLa steady state, whereas the remaining six rowers were unable to complete the test. In the subsequent study (7), carried out on 11 cyclists who performed a 30-min PET on a bicycle ergometer at power output corresponding to $\mathrm{HR}_{\mathrm{DP}}$, it was found that only four of them met the criteria for BLa steady state. The discrepancies in the results between these and our study may be partially explained by the training state of the subjects and by the variations in the accuracy of $\mathrm{HR}_{\mathrm{DP}}$ detection. Although all the runners in our study showed BLa steady state during the PET, it remains an unanswered question whether a $\mathrm{HR}_{\mathrm{DP}}$ reflects maximal lactate steady state (i.e., highest exercise intensity that can be maintained over time without continuous BLa accumulation), an issue that should be addressed in future studies.

A limitation of this study is the small sample size, which undoubtedly limits the generalization of the findings. However, the small sample size is a common characteristic of 
the majority of the previous $\operatorname{HR}_{\mathrm{DP}}$ studies $(7,8,17,18,22-24)$. Another limitation of this study is the fact that runners did not repeat the modified CT to assess reliability of this test.

\section{Conclusions}

The results of this study show that the modified CT accurately predicted both HR and RS at $\mathrm{LT}$ in the runners presenting a $\mathrm{HR}_{\mathrm{DP}}$. This suggests that the modified CT has a potential to be used as an alternative method for assessment of LT in distance runners. However, this study also shows that this test may not be suitable for all runners since $\mathrm{HR}_{\mathrm{DP}}$ is not always observable. Future research with greater sample size is required to substantiate our findings before conclusion can be drawn regarding the validity of the modified $\mathrm{CT}$.

\section{Acknowledgements}

We would like to thank Mr. Aleksandar Kiradjiev and Mr. Sasho Danevski for helping in recruiting the participants of this study. We would also like to thank Professor Snezana Stojanovska for proofreading the manuscript.

\section{REFERENCES}

1. Ballarin E, Borsetto C, Cellini M, Patracchini M, Vitiello P, Ziglio PG, Conconi F: Adaptation of the "Conconi test" to children and adolescents. Int. J. Sports Med. 10, 334-338 (1989)

2. Billat LV: Use of blood lactate measurements for prediction of exercise performance and for control of training. Recommendations for long-distance running. Sports Med. 22, 157-175 (1996)

3. Bishop D, Jenkins DG, Mackinnon LT: The relationship between plasma lactate parameters, Wpeak and 1-h cycling performance in women. Med. Sci. Sports Exerc. 30, 1270-1275 (1998)

4. Bodner ME, Rhodes EC: A review of the concept of the heart rate deflection point. Sports Med. 30, 31-46 (2000)

5. Bodner ME, Rhodes EC, Martin AD, Coutts KD: The relationship of the heart rate deflection point to the ventilatory threshold in trained cyclists. J. Strength Cond. Res. 16, 573-580 (2002)

6. Bourdon P (2013): Blood lactate transition thresholds: concepts and applications. In: Australian Sports Commission: Physiological Tests for Elite Athletes, 2nd ed., eds Tanner RK, Gore CJ, Human Kinetics, Champaign, IL, pp. 77-102

7. Bourgois J, Coorevits P, Danneels L, Witvrouw E, Cambier D, Vrijens J: Validity of the heart rate deflection point as a predictor of lactate threshold concepts during cycling. J. Strength Cond. Res. 18, 498-503 (2004)

8. Bourgois J, Vrijens J: The Conconi test: a controversial concept for the determination of the anaerobic threshold in young rowers. Int. J. Sports Med. 19, 553-559 (1998)

9. Bunc V, Hofmann P, Leitner H, Gaisl G: Verification of the heart rate threshold. Eur. J. Appl. Physiol. Occup. Physiol. 70, 263-269 (1995)

10. Cabo JV, Martinez-Camblor P, Del Valle M: Validity of the modified Conconi test for determining ventilatory threshold during on-water rowing. J. Sports Sci. Med. 10, 616-623 (2011)

11. Cellini M, Vitiello P, Nagliati A, Ziglio PG, Martinelli S, Ballarin E, Conconi F: Noninvasive determination of the anaerobic threshold in swimming. Int. J. Sports Med. 7, 347-351 (1986)

12. Cheng B, Kuipers H, Snyder AC, Keizer HA, Jeukendrup A, Hesselink M: A new approach for the determination of ventilatory and lactate thresholds. Int. J. Sports Med. 13, 518-522 (1992)

13. Conconi F, Ferrari M, Ziglio PG, Droghetti P, Codeca L: Determination of the anaerobic threshold by a noninvasive field test in runners. J. Appl. Physiol. Respir. Environ. Exerc. Physiol. 52, 869-873 (1982)

14. Conconi F, Grazzi G, Casoni I, Guglielmini C, Borsetto C, Ballarin E, Mazzoni G, Patracchini M, Manfredini F: The Conconi test: methodology after 12 years of application. Int. J. Sports Med. 17, 509-519 (1996)

15. Droghetti P, Borsetto C, Casoni I, Cellini M, Ferrari M, Paolini AR, Ziglio PG, Conconi F: Noninvasive determination of the anaerobic threshold in canoeing, cross-country skiing, cycling, roller, and ice-skating, rowing, and walking. Eur. J. Appl. Physiol. Occup. Physiol. 53, 299-303 (1985)

16. Erdogan A, Cetin C, Karatosun H, Baydar ML: Non-invasive indices for the estimation of the anaerobic threshold of oarsmen. J. Int. Med. Res. 38, 901-915 (2010) 
17. Fabre N, Passelergue P, Bouvard M, Perrey S: Comparison of heart rate deflection and ventilatory threshold during a field cross-country roller-skiing test. J. Strength Cond. Res. 22, 1977-1984 (2008)

18. Harrison J, Dawson B, Lanrence S, Blanskby B: Non-invasive and invasive determinations of the individual anaerobic threshold in competitive swimmers. J. Swim. Res. 8, 11-17 (1992)

19. Hofmann P, Bunc V, Leitner H, Pokan R, Gaisl G: Heart rate threshold related to lactate turn point and steadystate exercise on a cycle ergometer. Eur. J. Appl. Physiol. Occup. Physiol. 69, 132-139 (1994)

20. Hofmann P, Pokan R: Value of the application of the heart rate performance curve in sports. Int. J. Sports Physiol. Perform. 5, 437-447 (2010)

21. Jones AM: The physiology of the world record holder for the women's marathon. Int. J. Sports Sci. Coaching 1, 101-116 (2006)

22. Jones AM, Doust JH: The Conconi test in not valid for estimation of the lactate turnpoint in runners. J. Sports Sci. 15, 385-394 (1997)

23. Kuipers H, Keizer HA, de Vries T, van Rijthoven P, Wijts M: Comparison of heart rate as a non-invasive determinant of anaerobic threshold with the lactate threshold when cycling. Eur. J. Appl. Physiol. Occup. Physiol. 58, 303-306 (1988)

24. Lucía A, Carvajal A, Pérez M, Boraita A, Serratosa L, Chicharro JL: Heart rate response during incremental exercise in master runners. Jpn. J. Physiol. 50, 155-158 (2000)

25. Lucía A, Hoyos J, Santalla A, Pérez M, Carvajal A, Chicharro JL: Lactic acidosis, potassium, and the heart rate deflection point in professional road cyclists. Br. J. Sports Med. 36, 113-117 (2002)

26. McGehee JC, Tanner CJ, Houmard JA: A comparison of methods for estimating the lactate threshold. J. Strength Cond. Res. 19, 553-558 (2005)

27. Mikulic P, Vucetic V, Sentija D: Strong relationship between heart rate deflection point and ventilatory threshold in trained rowers. J. Strength Cond. Res. 25, 360-366 (2011)

28. Newell J, Higgins D, Madden N, Cruickshank J, Einbeck J, McMillan K, McDonald R: Software for calculating blood lactate endurance markers. J. Sports Sci. 25, 1403-1409 (2007)

29. Petit MA, Nelson CM, Rhodes EC: Comparison of a mathematical model to predict 10-km performance from the Conconi test and ventilatory threshold measurements. Can. J. Appl. Physiol. 22, 562-572 (1997)

30. Smith D, Telford R, Peltola E, Tumilty D (2000): Protocols for the physiological assessment of highperformance runners. In: Australian Sports Commission: Physiological Tests for Elite Athletes, ed Gore CJ, Human Kinetics, Champaign, IL, pp. 334-344

31. von Duvillard SP, Pokan R, Hofmann P, Wonish M, Smekal G, Beneke R, Leithäuser R: Comparing blood lactate values of three different handheld lactate analyzers to YSI 1500 lactate analyzer. Med. Sci. Sports Exerc. 37 , S25 (2005) 